\title{
ELECTOMYOGRAPHIC ANALYSIS OF THE ORAL PHASE OF SWALLOWING IN SUBJECTS REHABILITATED WITH MANDIBULAR IMPLANT RETAINED OVERDENTURE
}

\author{
Abdallah M. Ibrahim*
}

\begin{abstract}
Objective: The purpose of this study was to detect the impact of mandibular implant retained overdenture on electromyographic activity of submental muscles during swallowing in edentulous subjects.

Materials and methods: Fifteen completely edentulous subjects with problematic lower complete dentures were participated in this study. All subjects received a newly carefully constructed complete dentures and then the lower dentures were changed into mandibular implant retained overdentures by placing two implants on the mandibular canine regions and using ball attachments to retain the overdentures. All the subjects were submitted to surface electromyographic evaluation for the submental muscles without dentures, with complete dentures and with mandibular implant overdenture during swallowing of $10 \mathrm{ml}$ of water.
\end{abstract}

Results: There was a statistically significant increase in swallowing duration and electromyographic amplitude of submental muscles after complete dentures insertion and also after insertion of mandibular implant overdenture. Moreover, there was a statistically significant reduction in both swallowing duration and electromyographic amplitude of submental muscles for mandibular implant overdenture when compared to complete dentures. Percentage Overlapping Coefficient showed a non-significant differences between different oral conditions.

Conclusion: Rehabilitation of completely edentulous patient with mandibular implant retained overdenture revealed a reduction in swallowing duration and reduction in electromyographic amplitude of the submental muscles during swallowing.

\section{INTRODUCTION}

Complete denture wearers frequently complain from the mandibular denture concerning stability, retention and problems with oral functions. Instability of the mandibular denture lets maintaining the normal diet was difficult. ${ }^{(1)}$ Masticatory efficiency of the individual was affected by stability of the denture and the instability resulted in less uniform and irregular masticatory cycles. ${ }^{(2)}$ Moreover, the use of complete dentures affects the feeding, swallowing, and nutritional status of the individual. ${ }^{(3)}$

\footnotetext{
* Lecturer of Removable Prosthodontics, Faculty of Dentistry, Mansoura University, Egypt.
} 
Implant assisted overdenture is usually used for increasing the retention and stability of the complete denture..$^{(4)}$ Several previous study ${ }^{(5-7)}$ found high success rate regarding to placement of dental implants to attach an overdenture. Many studies ${ }^{(8-10)}$ reported that two implants retained mandibular overdenture opposed by maxillary complete denture is considered as the globally accepted treatment option. Different types of attachment systems are used with mandibular implant assisted overdenture for retaining or support the overdenture. ${ }^{(11)}$

Swallowing process is a complex neuromuscular function including coordination between the structure of oral cavity, pharynx, larynx, and esophagus. (12) The swallowing process was evaluated in four phases: oral preparatory phase, oral phase, pharyngeal phase, and esophageal phase. Oral preparatory and oral phases are under voluntary control while the pharyngeal and esophageal phases are under involuntary control. ${ }^{(13-15)}$

Swallowing process can be evaluated kinamatically ${ }^{(16)}$ or electromyographically. ${ }^{(17)}$ Surface electromyography (sEMG) represent the simplest method to evaluate the muscle activity included during swallowing process. ${ }^{(18,19)}$ During the oral phase of swallowing, the muscles of mastication (temporalis, pterygoid, and masseter muscles) and the opening muscles (submental muscles) stabilize the mandible during oral closure and tongue elevation. ${ }^{(18,20)}$ During swallowing in the edentulous subjects the tongue was placed forward between the maxillary and mandibular edentulous ridges to make a seal. (21) The placement of the tongue between the two edentulous ridges during swallowing for edentulous subject was considered as a physiological process. Furthermore, swallowing without occlusal contact is considered as atypical swallowing and need treatments. ${ }^{(22)}$

Measuring swallowing duration is very important because prolonged swallowing duration cause high risk of laryngeal aspiration. ${ }^{(23)}$ The normal swallowing duration is attained at the 12 years of age and does not significantly changed until the age of 70 years, then with increasing the age more than 70 years the swallowing duration was significantly increased. ${ }^{(22,24)}$

Yoshikawa et al., ${ }^{(25)}$ studied the swallowing function for completely edentulous subjects with cinefluorography and reported that there was significant reduction in oral transit duration for edentulous subject not wearing complete dentures. Kodaira et al., ${ }^{(26)}$ studied the impact of palatal mucosa covering by denture base on swallowing function and found that covering the palatal mucosa by the denture base affect the swallowing duration.

Tallgren et al., ${ }^{(27)}$ investigated the surface electromyography of masseter, anterior temporalis, and lip muscles during swallowing in denture wearers. They found that the swallowing durations for water were significantly increased compared to complete natural dentition. Also, Tallgren and Tryde $^{(28)}$ reported that in patient wearing upper complete denture and lower partial denture, the swallowing duration was significantly longer after one year of dentures wearing, due to bone resorption and tissue changes, these might cause impairment of denture stability leading to increase in swallowing duration. Monaco et al. ${ }^{(29)}$ reported that swallowing duration for spontaneous saliva swallowing for complete denture wearer was correlated to the quality of denture fit. Swallowing duration for poorly fitted complete denture was significantly longer than for the well fitted complete denture.

Masticatory function was affected by the lack of stability of the complete denture. ${ }^{(30)}$ Implant overdenture could improve the oral function and the masticatory performance as it improve the stability of the overdenture ${ }^{(31)}$ that allow a regular masticatory cycles and a higher electrical activity of masseter muscles compared to conventional complete denture. ${ }^{(32)}$ The role of mandibular implant retained overdenture in function of mastication was well 
established but the impact of mandibular implant retained overdenture on swallowing function has not been fully analyzed. The purpose of the current study was to evaluate the effect of mandibular implant retained overdenture on oral phase of swallowing using surface electromyographic analysis.

\section{MATERIALS AND METHODS}

Fifteen healthy completely edentulous subjects were participated in this study. They were selected from Outpatients Clinic, Prosthodontics Department, Faculty of Dentistry, Mansoura University. Their ages ranged from 50 to 65 years. All subjects were complete denture wearers presented to the clinic with chronic problems particularly in respect of instability and discomfort of the mandibular dentures. All subjects were given an idea about the nature of this research work and the proposed implant treatment plan. Their approvals were obtained, and the study protocol approval was taken from ethical committee. All subjects were nonsmokers, free from any systemic diseases that interfere with implant placement, free from any medical or surgical history of swallowing problems and had normal maxillo-mandibular relationship.

\section{Surgical and prosthetic procedures}

All subjects were received newly constructed complete dentures with optimal denture flanges extension and well established lingualized balanced occlusion. The lower dentures were duplicated into clear acrylic resin duplicates. The dentures duplicates were modified as a radiographic stent by preparing multiple holes $(2 \mathrm{~mm}$ depth and $2 \mathrm{~mm}$ width) filled with radiopaque gutta-percha in order to act as reference points during the construction of stereolithographic surgical guide and determine the amount of bone for implant placement. ${ }^{(33)}$

Stereolithographic mucosal supported surgical guide was constructed after dual scan technique using cone beam computerized tomography (CBCT, i- CAT Vision ${ }^{\circledR}$, Imaging Sciences International, Hatfield, PA). Two dental implants (Dyna Dental Engineering, Bergen op Zoom, the Netherlands) were placed in the mandibular ridge in the canine regions by means of guided surgery using submerged surgical technique and delayed loading protocol. Prophylactic antibiotic (875 mg Amoxicillin and Clavulanic acid $125 \mathrm{mg}$ ) was taken one day before implant placement and continued for one week after surgery. The subjects were informed to rinse their mouth with chlorohexidine mouthwash just before surgery and continue rinsing their mouth one week after surgery.

Immediately after surgery, no prosthesis should be used over the implant surgical site. After one week, the sutures were removed, and the intaglio surface of the existing mandibular denture was relieved in the area overlying the implants and relined using tissue conditioning material (alphadent DENTAL TECHNOLOGIES. USA) then the denture was delivered to the patient and follow up visits were scheduled.

After the period of osseo-integration (3-4 months), ball attachments (Ball Abutment and Gold Smart Matrix, Dyna Dental Engineering) were used to attach the implant to the overdenture. The implant was exposed by using tissue punch and healing abutment were placed. After one week the healing abutment were replaced by ball abutment (Fig. 1). The mandibular complete denture was converted to mandibular implant retained overdenture by incorporating the housings of the ball attachments to the intaglio surface of the lower denture by using direct pick-up technique (Fig. 2).

\section{Electromyographic analysis}

Surface electromyographic (sEMG) evaluation for the subjects was carried out after one month of denture or overdenture delivery and performed at three different oral conditions:

- Without denture. 
- With maxillary and mandibular complete dentures.

- With mandibular implant retained overdenture opposed by maxillary complete denture.

Surface electromyographic activity of the submental muscles was recorded in order to analyze the activation pattern of the submental muscles bilaterally and to visualize the activation pattern of the muscles during swallowing function. Surface electromyography electrodes (disposable active, pre-gelled, silver, or silver chloride bipolar electrode) were attached to the area of submental muscles (right and left) by using adhesive bandage tape. The electrodes were placed in the submental area parallel to the direction of the muscle fibers (involving anterior belly of digastric, mylohyoid and geniohyoid muscles), para-median to the midline, lightly divergent and $1 \mathrm{~cm}$ posterior to the mental symphysis. ${ }^{(34)}$ The skin was firstly cleaned with alcohol before placement of electrodes to prevent skin impedance and allowing the conductive past to adequately moisten the skin. Another electrode was attached to the skin of the forehead area and considered as a ground electrode. The recording electrodes were connected to computerized measuring system (NihonNKohden Corp., Tokyo, Japan). Surface electromyographic signals were digitized, amplified, and sampled at $1500 \mathrm{HZ}$. The sEMG signals were full rectified, filtered and smoothened. The signals were averaged over $25 \mathrm{~ms}$

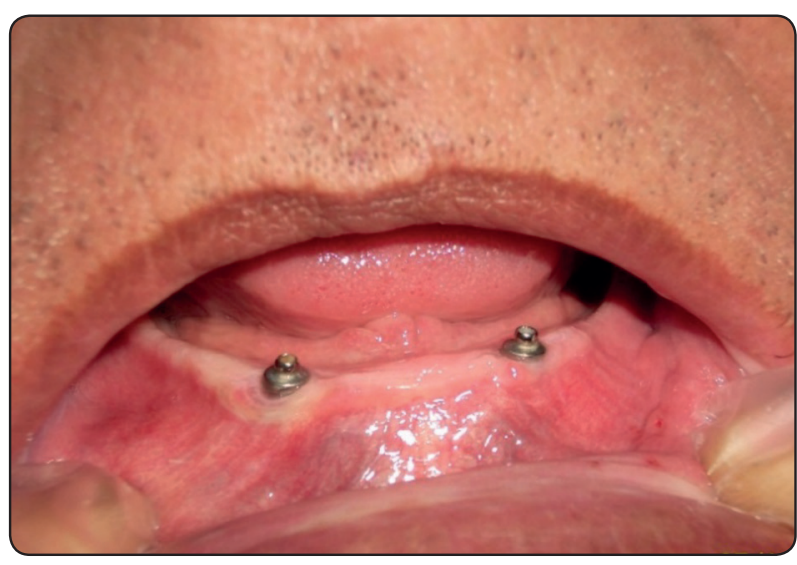

Fig. (1) Ball abutment secured to the dental implant and the muscle activity recorded as the amplitude (mVolt) (Fig .3) then sEMG signals were recorded for further analysis.

sEMG recording was carried out during swallowing of $10 \mathrm{~mL}$ of water and 5 seconds was recorded. During the test, the subjects were set comfortably in an office chair with straight posture and the feet were flat on the floor and the arms were relaxed on their legs and the subjects do their best to avoid head movement.

From the sEMG wave, the following further measurements were recorded:

1- Percentage Overlapping Coefficient (POC\%): Symmetry of the muscles evaluated by calculating the percentage overlapping coefficient ${ }^{(35)}$, the index of symmetry is ranging from $0 \%$ to $100 \%$. When two paired of muscles contract with perfect symmetry a POC $\%$ of $100 \%$ is achieved.

2- Swallowing Duration: The interval between the beginning of first muscle activation to the end of the last muscle activation.

\section{Statistical analysis}

Statistical analyses were preformed using the statistical package for social scientists (SPSS) for windows version 20.0 program. To test for normal distribution frequency of data was plotted against normal distribution curve, non-parametric statistical

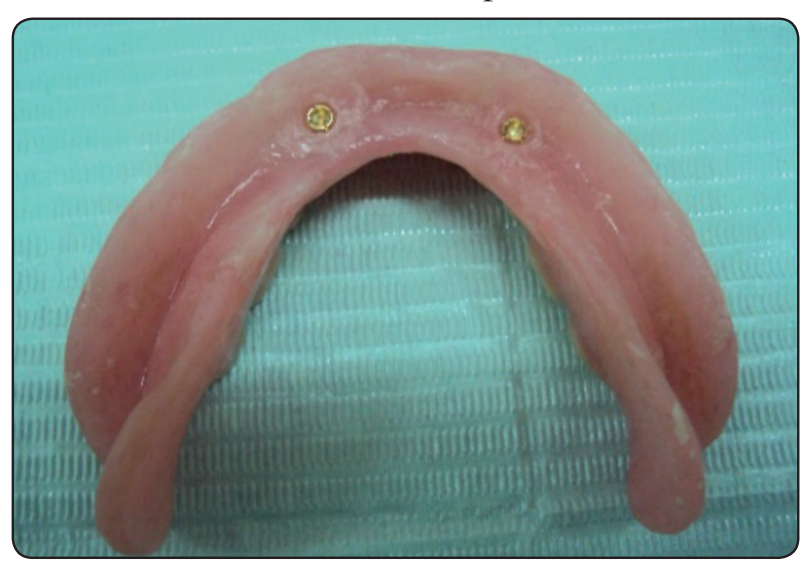

Fig. (2): The overdenture after pick-up the female housing of the ball attachment 


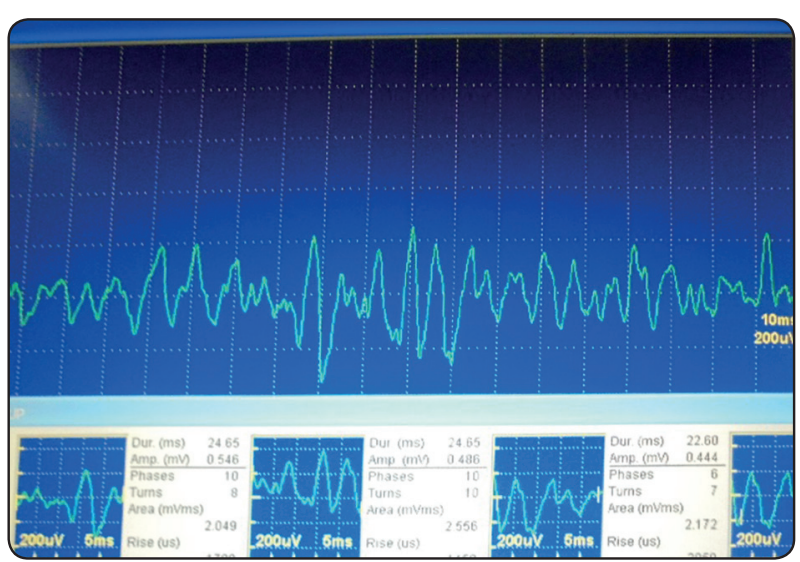

Fig. (3): An example sEMG signal averaged over $25 \mathrm{~ms}$

methods were used to test for significance of change, Wilcoxon signed rank test was used to compare quantitative data while Mc-Nemar test of change was used to compare qualitative data. $\mathrm{P}$ value was considered significant if less than 0.05 .

\section{RESULTS}

Table (1) showed the descriptive analysis of surface electromyography of submental muscles during swallowing of $10 \mathrm{ml}$ of water for different oral conditions (without denture, with complete dentures, and with mandibular implant overdenture). The means of swallowing durations (per second) for without denture, with complete dentures, and with mandibular implant overdenture were $(1.42 \pm 0.1$, $1.81 \pm 0.13,1.55 \pm 0.12)$ respectively. The means of
sEMG amplitudes $(\mathrm{mV})$ for without denture, with complete dentures, and with mandibular implant overdenture for the right side were $(0.398 \pm 0.12$, $0.559 \pm 0.23,0.462 \pm 0.16)$ respectively, and for the left side were $(0.375 \pm 0.09,0.540 \pm 0.10$, $0.486 \pm 0.11$ ) respectively. Also, the means of Percentage Overlapping Coefficient (POC \%) for without denture, with complete dentures and with mandibular implant overdenture were $(83.3 \pm 6.4$, $84.1 \pm 8.5,84.2 \pm 7.5$ ) respectively.

Table (2) showed the comparison between different oral conditions for swallowing duration, sEMG amplitude, and POC\% for submental muscles during swallowing. There was statistically significant reduction of swallowing duration for without denture when compared to both complete dentures and mandibular implant overdenture. Also, there was statistically significant reduction of swallowing duration for mandibular implant overdenture when compared to complete dentures.

For sEMG amplitude, without denture showed statistically significant reduction when compared to the other different oral conditions (for both right and left sides). Also, there was statistically significant reduction of sEMG amplitude for mandibular implant overdenture when compared to complete dentures oral condition (for both right and left sides).

Regarding to $\mathrm{POC} \%$ there was no significant differences between different oral conditions.

TABLE (1): Descriptive statistical analysis for swallowing duration, sEMG amplitude and POC\% for without denture, with complete dentures, and with mandibular implant overdenture .

\begin{tabular}{|c|c|c|c|c|}
\hline \multirow{2}{*}{} & Without dentures & Complete dentures & Implant overdenture \\
\cline { 2 - 4 } & Mean \pm SD & Mean \pm SD & Mean \pm SD \\
\hline \multicolumn{2}{|c|}{ Swallowing duration $(\mathbf{s})$} & $1.42 \pm 0.1$ & $1.81 \pm 0.13$ & $1.55 \pm 0.12$ \\
\hline \multirow{2}{*}{ SEMG amplitude (mV) } & RT & $0.398 \pm 0.12$ & $0.559 \pm 0.23$ & $0.462 \pm 0.16$ \\
\cline { 2 - 5 } & LF & $0.375 \pm 0.09$ & $0.540 \pm 0.10$ & $0.486 \pm 0.11$ \\
\hline \multicolumn{2}{|c|}{ POC\% } & $83.3 \pm 6.4$ & $84.1 \pm 8.5$ & $84.2 \pm 7.5$ \\
\hline
\end{tabular}


TABLE (2): Comparison of the means for swallowing duration, sEMG amplitude, and POC\% between different oral conditions (without denture, with complete dentures, with mandibular implant overdenture).

\begin{tabular}{|c|c|c|c|}
\hline \multicolumn{3}{|c|}{ Comparison between different oral conditions } & P value \\
\hline \multirow{3}{*}{\multicolumn{2}{|c|}{ Swallowing duration }} & Without denture \&Complete dentures & 0.005 \\
\hline & & Without denture \&Implant overdenture & 0.009 \\
\hline & & Complete dentures \&Implant overdenture & 0.01 \\
\hline \multirow[t]{6}{*}{ sEMG amplitude } & \multirow[t]{3}{*}{ RT } & Without denture \&Complete dentures & 0.003 \\
\hline & & Without denture \&Implant overdenture & 0.002 \\
\hline & & Complete dentures \&Implant overdenture & 0.03 \\
\hline & \multirow[t]{3}{*}{$\mathbf{L F}$} & Without denture \&Complete dentures & 0.005 \\
\hline & & Without denture \&Implant overdenture & 0.004 \\
\hline & & Complete dentures \&Implant overdenture & 0.02 \\
\hline \multirow{3}{*}{\multicolumn{2}{|c|}{ POC\% }} & Without denture \&Complete dentures & 0.08 \\
\hline & & Without denture \&Implant overdenture & 0.09 \\
\hline & & Complete dentures \&Implant overdenture & 0.11 \\
\hline
\end{tabular}

RT: right side

LF : left side

\section{DISCUSSION}

Total edentulism or loss of occlusal contact led to atypical swallowing, the solution for this problem is to re-establish the occlusion with dental prosthesis. $^{(22)}$ kondoh et al et al., ${ }^{(36)}$ studied the effect of wearing complete dentures on swallowing function by pressure sensor placed on denture palate. They found that there was a confusion between oral structure and the pharynx when the patient swallowed without wearing the dentures and the occlusal contact occur during swallowing after wearing the complete denture was supposed to make sequential coordination among the muscles and reported that swallowing function was deteriorated because of the reduction in tongue palatal contact on removing the complete dentures.

The present study showed reduction in swallowing duration for without denture oral condition when compared to both complete dentures and mandibular implant overdenture. This may be due to the complete dentures or the conventional implant overdentures occupy a large space intraorally and the palatal mucosa is covered by the palatal part of maxillary denture. This was in agreement with Gokce et al., ${ }^{(37)}$ they found that swallowing time was greatly decreased for edentulous subjects than after wearing the complete dentures. Kodaira et al., (26) also reported that the thickness of the complete dentures might interfere with the sensation of the tongue and then the longest swallowing duration was presented in compete denture wearer when compared with without denture.

Moreover, a greater sEMG amplitude of submental muscles was found after wearing the complete dentures. This might be explained by the involvement of larger number of motor units to retain the mandibular removable denture. Veyrune and Mioche ${ }^{(38)}$ reported that teeth loss and wearing a complete denture affect the motor and sensory units involved during oral functions.

The result of this study showed a reduction in swallowing duration for mandibular implant over- 
dentures when compared to complete dentures oral condition. This could be explained by the implant enhance the retention and stability of the overdentures leading to a more comfort and high confidence during swallowing. This was in agreement with Monaco et al. ${ }^{(29)}$ they concluded that well fitted denture reduce the swallowing duration toward the physiological value. Also, mandibular implant overdenture showed statistically significant reduction in sEMG amplitude in submental muscles when compared to after wearing complete dentures. This might be due to adaptation of the stomatognathic system to the oral conditions of the individuals achieved after stabilization of the overdenture by implant placement inducing a reduction in muscular activity. This was in accordance with Beretin-Felix et al., ${ }^{(39)}$ study that found a reduction in bioelectric activity of submental muscles during swallowing after oral rehabilitation with fixed implant supported prosthesis.

Normally, when POC\% index is about $80 \%$ $85 \%$ a good symmetrical activation of each pairs of muscles is presented. ${ }^{(35)}$ In the current study, a higher $\mathrm{POC} \%$ value for different oral conditions were presented, as the floor of the mouth was formed of overlapping muscular fibers ${ }^{(40)}$ and during swallowing, the submental muscles were activated with the movement of the tongue against the palate, to firming the floor of the mouth. ${ }^{(34)}$ Similarly, Dellavia et al., ${ }^{(17)}$ recorded a high values of symmetry of submental muscles during swallowing.

Previous researches reported that swallowing duration affected by the volume or the consistency of the swallowed bolus. ${ }^{(41,42)}$ Moreover, to study the swallowing function during swallowing solid foods for complete denture wearers, several factors must be examined such as: condition of residual ridges, form of denture base, artificial teeth position, habitual masticatory side, period of denture adaptation, and so on. ${ }^{(25)}$ So in the present study, $10 \mathrm{ml}$ of water was used as standardized bolus to establish basic data and the results were simply evaluated.

\section{CONCLUSIONS}

Within the limitation of this study, it may be concluded that:

Rehabilitation of completely edentulous patient with mandibular implant retained overdenture revealed a reduction in swallowing duration toward the physiological value and also reduction of the electromyographic amplitude of the submental muscles during water swallowing. This may indicate the adaptation to the new condition of retention and stability provided by the mandibular implant retained overdenture.

\section{REFERENCES}

1. Van Kampen FMC, Van der Bilt A, Cune MS, FontijnTekamp FA, Bosman F. Masticatory function with implant supported overdentures. J Dent Res. 2004, 83: 708-711.

2. Karkazis HC, Kossioni AE. Surface EMG activity of the masseter muscle in denture wearers during chewing of hard and soft food. J Oral Rehabil. 1998, 25:8-14.

3. Ship JA, Duffy V, Jones JA, Langmore S. Geriatric oral health and its impact on eating. J Am Geriatr Soc. 1996, 44:456-64.

4. Cune MS, van Kampen FMC, van der Bilt A et al. Patient satisfaction and preference with magnet, bar-clip, and ball socket retained mandibular implant overdentures: a crossover clinical trial. J Prosthodont. 2005, 18: 99-105.

5. Payne AG, Tawes-Smith A, Kumara R and Thomson WM. One-year prospective evaluation of the early loading of unsplinted conical branemark fixtures with mandibular overdentures immediately following surgery. Clin. Implant Dent. Relat. Res. 2001, 3: 9-19.

6. Chiapasco M and Gatti C. Implant retained mandibular overdentures with immediate loading: A 3-to 8-year prospective study on 328 implants. Clin Implant Dent. Res. 2003, 5: 29-38.

7. Stephan G, Vidot F, Noharet R and Mariani P. Implant retained mandibular overdentures: A comparative pilot study of immediate loading versus delayed loading after two years. J Prosthet. Dent. 2007, 97: 38-45.

8. Naert I, Alsaadi G, Quirynen M. Prosthetic aspects and patient satisfaction with two-implant-retained mandibular 
overdentures: a 10-year randomized clinical study. Int J Prosthodont. 2004, 17: 401-10.

9. Visser A, Meijer HJ, Raghoebar GM, Vissink A. Implant retained mandibular overdentures versus conventional dentures: 10 years of care and aftercare. Int J Prosthodont. 2006, 19:271-8.

10. Meijer HJ, Raghoebar GM, Batenburg RH, Visser A, Vissink A. Mandibular overdentures supported by two or four endosseous implants: a 10-year clinical trial. Clin Oral Implants Res. 2009, 20:722-8.

11. Besimo CH, Graber G, Fluhler M. Retention force changes in implant-supported titanium telescope crowns over long term use in vitro. J Oral Rehabil. 1996, 23:372.

12. Honda Y, Hata N. Dynamic imaging of swallowing in a seated position using open-configuration MRI. J Magn Reson Imaging. 2007, 26:172-176.

13. Furia CL, Carrara-de-Angelis E, Martins NM, Barros AP, Carceiro B, Kowalski LP. Videofluoroscopic evaluation after glossectomy. Arch Otolaryngol Head Neck Surg. 2000, 126: 378-383.

14. Anagnostara A, Stoeckli S, Weber OM, Kollias SS. Evaluation of the anatomical and functional properties of deglutition with various kinetic high-speed MRI sequences. J Magn Reson Imaging. 2001, 14:194-199.

15. Hartl DM, Albiter M, Kolb F, Luboinski B, Sigal R. Morphologic parameters of normal swallowing events using single shot fast spin echo dynamic MRI. Dysphagia. 2003, 18: 255-262.

16. Pasinato F, Oliveira AG, Santos-Couto-Paz CC, et al. Study of the kinematic variables of unilateral and habitual mastication of healthy individuals. Codas. 2017, 29:e20160074.

17. Dellavia C, Rosati R, Musto F, Pellegrini G, Begnoni G, Ferrario VF. Preliminary approach for the surface electromyographical evaluation of the oral phase of swallowing. J Oral Rehabil. 2018, 45:518-525.

18. Vaiman M, Eviatar E, Segal S. Surface electromyographic studies of swallowing in normal subjects: a review of 440 adults. Report 3. Qualitative data. Otolaryngol Head Neck Surg. 2004, 131: 977-985.

19. Vaiman M. Standardization of surface electromyography utilized to evaluate patients with dysphagia. Head Face Med. 2007, 3:26.
20. Ertekin C, Aydogdu I. Neurophysiology of swallowing. Clin Neurophysiol. 2003, 114:2226-2244.

21. Yoshikawa M, Yoshida M, Nagasaki T, Tanimoto K, Tsuga $\mathrm{K}$, Akagawa Y. Effects of tooth loss and denture wear on tongue-tip motion in elderly dentulous and edentulous people. J Oral Rehabil. 2008, 35:882-888.

22. Monaco A, Cattaneo R, Spadaro A, Giannoni M. Surface electromyography pattern of human swallowing. BMC Oral Health. 2008, 8:6.

23. Leonard R, McKenzie S. Hyoid-bolus transit latencies in normal swallow. Dysphagia. 2006, 21:183-190.

24. Vaiman M, Eviatar E, Segal S. Evaluation of normal deglutition with the help of rectified surface electromyography. Dysphagia. 2004, 19:125-132.

25. Yoshikawa M, Yoshida M, Nagasaki T, Tanimoto K, Tsuga $\mathrm{K}$, Akagawa Y. Influence of aging and denture use on liquid swallowing in healthy dentulous and edentulous older people. J Am Geriatr Soc. 2006, 54:444-449.

26. Kodaira Y, Ishizaki K, Sakurai S. Effect of palate covering on bolus-propulsion time and its contributory factors. J Oral Rehabil. 2006, 33:8-16.

27. Tallgren A, Lang BR, Holden S, Miller RL. Longitudinal electromyographic study of swallowing patterns in complete denture wearers. Int J Prosthodont 1995, 8: 467-478.

28. Tallgren A, Tryde G. Swallowing activity of lip muscles in patients with complete upper and partial lower denture. J Oral Rehabil 1992, 19: 329-341.

29. Monaco A, Cattaneo R, Masci C, Spadaro A, Marzo G. Effect of ill-fitting dentures on the swallowing duration in patients using polygraphy. Gerodontology 2012, 29: e637-e644

30. Fujimori T, Hiraano S, Hayakawa I. Effects of a denture adhesive on mastication functions for complete denture wearers. J Med Dent Sci. 2002, 49: 151-156.

31. Van der Bilt A, Van Kampen FMC, Cune MS. Masticatory function with mandibular implant supported overdentures fitted with different attachment types. Eur J Oral Sci. 2006, 114: 191- 196 .

32. Karkazis HC. EMG activity of the massager muscle in implant supported overdenture wearers during chewing of hard and soft food. J. Oral Rehabil. 2002, 29:986-991.

33. Abdelhamid AM, Hanno KI, Imam MH. A prospective cross-over study to evaluate the effect of two different 
occlusal concepts on the masseter muscle activity in implant-retained mandibular overdentures. Int $\mathbf{J}$ Implant Dent 2015, 1: 32 .

34. Musto F, Rosati R, Sforza C, Toma M, Dellavia C. Standardised surface electromyography allows effective submental muscles assessment. J Electromyogr Kinesiol. 2017, 34:1-5.

35. Ferrario VF, Sforza C, Colombo A, Ciusa V. An electromyographic investigation of masticatory muscles symmetry in normo-occlusion subjects. J. Oral Rehabil. 2000, 27:33-40.

36. Kondoh J, Ono T, Tamine K, Fujiwara S, Minagi Y, Hori K, Maeda Y, Kreissl M and Nitschke I. Effect of complete denture wearing on tongue motor biomechanics during swallowing in edentulous older adults. Geriatr Gerontol Int 2015, 15: 565-571

37. Gokce HS, Gokce SM, Akin E, Bulakbasi N, Akyol M. Effect of complete denture wearing on deglutition time: a cine-magnetic resonance imaging study. J. Oral Rehabil. 2012, 39: 198-209.
38. Veyrune JL, Mioche L. Complete denture wears: electromyography of mastication and texture perception whilst eating meat. Eur J Oral Sci. 2000, 108:83-92.

39. Berretin-felix G, Nary Filho H, padovani CR, Trindade Junior AS, Machado WM. Electromyographic evaluation of mastication and swallowing in elderly individuals with mandibular fixed implant supported prostheses. J Appl Oral Sci. 2008, 16:116-21.

40. Ferrario VF, Sforza C, D'addona A, Miani Jr A. Reproducibility of electromyographic measures: a statistical analysis. J Oral Rehabil. 1991, 18:513-521.

41. Inagaki D, Miyaoka Y, Ashida I, Yamada Y. Influence of food properties and body posture on durations of swallowing-related muscle activities. J Oral Rehabil. 2008, 35: 656-663.

42. Karacay S, Akin E, Sayin MO, Bulakbasi N. Real time balanced turbo field echo(b-tfe) cine-MRI in the analysis of deglutition events and transit times. J Oral Rehabil. 2006, 33: 646-653 